\title{
Soil-transmitted helminthiasis in four districts in Bangladesh: household cluster surveys of prevalence and intervention status
}

Stacy L. Davlin ${ }^{1 \dagger}$, Alexander H. Jones ${ }^{1 \dagger}$ (D) Sanya Tahmina², Abdullah Al Kawsar ${ }^{3}$, Anand Joshi ${ }^{1}$, Sazid I. Zaman ${ }^{4}$, Muhammad M. Rahman ${ }^{3}$, Bozena M. Morawski ${ }^{1}$, Michael S. Deming ${ }^{1}$, Rubina Imtiaz ${ }^{1}$ and Mohammad J. Karim ${ }^{3^{*}}$

\begin{abstract}
Background: In 2016, after 8 years of twice-annual nationwide preventive chemotherapy (PC) administration to school-age children (SAC), the Bangladesh Ministry of Health \& Family Welfare (MOHFW) sought improved impact and intervention monitoring data to assess progress toward the newly adopted goal of eliminating soil-transmitted helminthiasis (STH) as a public health problem.

Methods: We surveyed four Bangladeshi districts between August and October 2017. We conducted a multi-stage, cluster-sample, household survey which produced equal-probability samples for preschool-age children (PSAC; 1-4 years), SAC (5-14 years), and adults ( $\geq 15$ years). Standardized questionnaires were administered, using Androidbased smart phones running an Open Data Kit application. Stool samples were collected and testing for STH prevalence and infection intensity used the Kato-Katz technique.

Results: In all, 4318 stool samples were collected from 7164 participants. Estimates of STH prevalence by risk group in three of the four surveyed districts ranged from 3.4 to 5.0\%, all with upper, 1-sided 95\% confidence limits $<10 \%$. However, STH prevalence estimates in Sirajganj District ranged from 23.4 to 29.1\%. Infections in that district were spatially focal; four of the 30 survey clusters had $>50 \%$ prevalence in at least one risk group. Among all tested specimens, Ascaris lumbricoides was the most common STH parasite [8.2\% $(n=352)]$, followed by Trichuris trichiura [0.9\% $(n=37)]$, and hookworm [0.6\% $(n=27)]$. In each district, PC coverage among SAC was above the 75\% program target but did not exceed 45\% among PSAC in any district. Improved sanitation at home, school, or work was over $90 \%$ in all districts.

* Correspondence: jahirulkarim@gmail.com

${ }^{\dagger}$ Stacy L. Davlin and Alexander H. Jones contributed equally to this work.

${ }^{3}$ Elimination of Lymphatic Filariasis \& STH Control Program, Directorate

General of Health Services, Ministry of Health \& Family Welfare, Road \# 29,

New DOHS, Mohakhali, Dhaka, Bangladesh

Full list of author information is available at the end of the article

\section{$\triangle B M C$}

(c) The Author(s). 2020 Open Access This article is licensed under a Creative Commons Attribution 4.0 International License, which permits use, sharing, adaptation, distribution and reproduction in any medium or format, as long as you give appropriate credit to the original author(s) and the source, provide a link to the Creative Commons licence, and indicate if changes were made. The images or other third party material in this article are included in the article's Creative Commons licence, unless indicated otherwise in a credit line to the material. If material is not included in the article's Creative Commons licence and your intended use is not permitted by statutory regulation or exceeds the permitted use, you will need to obtain permission directly from the copyright holder. To view a copy of this licence, visit http://creativecommons.org/licenses/by/4.0/ The Creative Commons Public Domain Dedication waiver (http://creativecommons.org/publicdomain/zero/1.0/) applies to the data made available in this article, unless otherwise stated in a credit line to the data. 


\begin{abstract}
(Continued from previous page)
Conclusions: In the three low-prevalence districts, the MOHFW is considering decreasing the frequency of mass drug administration, per World Health Organization (WHO) guidelines. Also, the MOHFW will focus programmatic resources and supervisory efforts on Sirajganj District. Despite considering WHO guidance, the MOHFW will not expand PC administration to women of reproductive age partly due to the low prevalence of hookworm and $T$. trichiura, the STH parasites that contribute most to morbidity in that risk group. Data collected from surveys such as ours would help effectively guide future STH control efforts in Bangladesh and elsewhere.
\end{abstract}

Keywords: Soil-transmitted helminthiasis, Epidemiology, Integrated survey, Preventive chemotherapy, Water, sanitation, and hygiene, Bangladesh

\section{Background}

Over one billion people in more than 100 countries have soil-transmitted helminthiasis (STH) [1] which is caused by the parasites Ascaris lumbricoides, Trichuris trichiura, and the hookworm species, Necator americanus and Ancylostoma duodenale. The World Health Organization (WHO) has established the goal of elimination of STH as a public health problem by 2020 , defined as $<1 \%$ moderate-to-high intensity infection (MHII) in at-risk preschool-age children (PSAC; 1-4 years old) and school-age children (SAC; 5-14 years old) [2]. Women of reproductive age (WRA; 15-49 years old) are another WHO-identified risk group [3].

Many national STH control programs, including in Bangladesh, have struggled to demonstrate progress toward elimination of STH as a public health problem. Additionally, national programs often rely on schoolbased preventive chemotherapy (PC) targeting SAC as the primary intervention to achieve the program goal. PSAC and WRA receive PC through different platforms, if at all. Bangladesh and other national programs use PC coverage as the primary indicator of success. While important, PC coverage does not directly evaluate impact or the prevalence thresholds used to determine mass drug administration (MDA) frequency [4]. Additionally, many national programs rely on the WHO methodology for school-based parasitologic surveys to monitor prevalence [5]. Given species-specific epidemiology and drug sensitivity, parasitologic assessment of SAC alone may not accurately represent $\mathrm{STH}$ epidemiology in all risk groups.

In 2005 and 2007, the Bangladesh Ministry of Health \& Family Welfare (MOHFW) conducted baseline schoolbased STH parasitologic surveys [5] in 5 of 64 districts and found that $80.0 \%$ of surveyed school children were STH positive. Consequently, the MOHFW initiated a nationwide STH control program in 2008 and has since conducted twice-annual MDA among SAC. By April 2018, the program completed 20 nationwide rounds of MDA using mebendazole. Additionally, 19 districts received annual community-wide MDA for lymphatic filariasis (LF). Since 2013, the MOHFW has reported $>75 \%$ annual national PC coverage for SAC [6] and currently targets 40.6 million children. The MOHFW adopted the goal of eliminating STH as a public health problem among children in 2016, which necessitated increased program monitoring. To assess progress toward the newly adopted goal, the MOHFW and Children Without Worms developed the "Integrated Community-based Survey for Program Monitoring" (ICSPM).

Based on a standardized protocol, the ICSPM provides statistically valid, district-level estimates of STH and parasite-specific prevalence and intensity of infection for each risk group; evaluates MOHFW-reported PC coverage; and measures sanitation coverage and specific hygiene-related behaviors. This publication presents the results of four ICSPM surveys and discusses their programmatic implications. There are no baseline data from the surveyed districts. Further evaluation of the ICSPM methodology is planned with the hope that the methodology will benefit other national programs that have the same programmatic goal.

\section{Methods}

\section{Study setting and population}

We surveyed Joypurhat, Chapai Nawabganj, Sirajganj, and Rajshahi districts (Fig. 1). Joypurhat has never received LF interventions. Chapai Nawabganj, Sirajganj, and Rajshahi districts received multiple years of LF MDA before stopping in 2013, 2012, and 2011, respectively [7]. District population size ranges from 1.0 million (Joypurhat) to 3.1 million (Sirajganj). The population is predominantly rural (range from $69 \%$ to $86 \%$ ). The districts were selected because they were participating in a joint STH-nutrition project, which required baseline assessment. We sampled the WHO risk groups: PSAC (14 years old) and SAC (5-14 years old). The MOHFW also decided to sample all adults ( $\geq 15$ years old).

\section{Ethical considerations}

The survey protocol was approved by the Bangladesh Medical Research Council. Participation in the survey was voluntary. Informed consent was obtained from all participants $\geq 18$ years old. Enrollment of participants < 18 years old required consent from a parent or guardian 


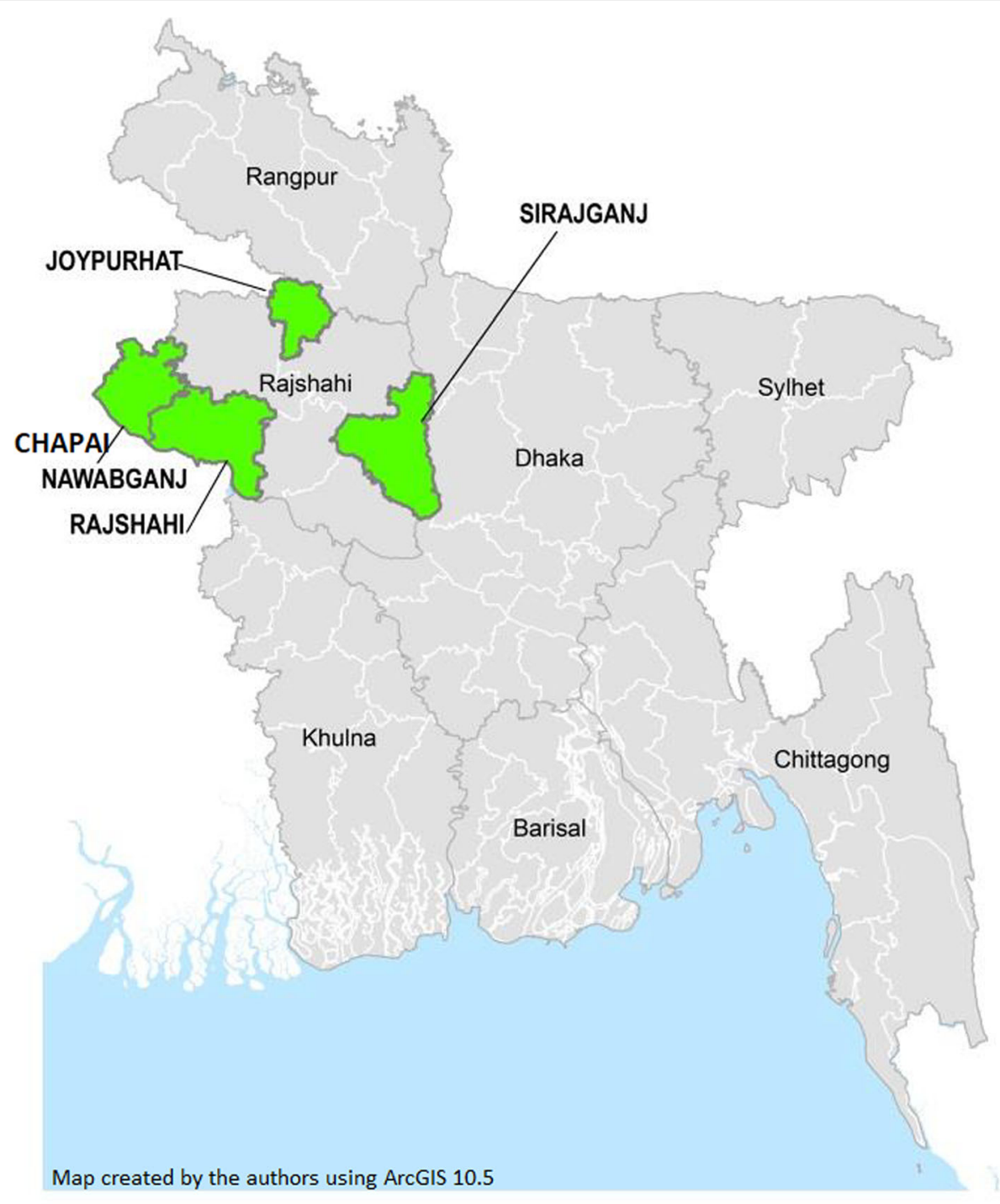

Fig. 1 Districts surveyed, Rajshahi Division, Bangladesh, August - October 2017

and assent from any person 5-17 years old. Participants 1-4 years old were enrolled with consent of a parent or guardian.

\section{Study design and data collection}

The ICSPM is a multi-stage, cluster-sample household survey. It expands the WHO Assessing the epidemiology of STH during a TAS module [4] by including all WHOidentified STH risk groups rather than SAC only. It modifies the sampling design of the WHO Transmission Assessment Survey for LF Elimination by adding segmentation, which improves efficiency of household selection in the field [8].

The ICSPM facilitates classification of each STH risk group according to WHO-defined prevalence ranges [5]. Risk group prevalence is classified by calculating the upper, 1-sided 95\% confidence limit. The prevalence range in which this limit falls is the lowest range in which there can be $95 \%$ confidence that true prevalence lies. The target sample size of 332 persons per risk group is adequate for determining if a $<10 \%$ prevalence threshold has been achieved and for higher thresholds. Details of this approach are discussed elsewhere [9].

The sample design provides an equal-probability sample from each risk group in each district. Villages were used as primary sampling units as data on census enumeration areas were unavailable. The number of households in a village was estimated based on 2011 national census data adjusted for population growth [10]. In all, 30 villages (clusters) were selected using probability proportional to their size in 100-household segments. In the field, these villages were subdivided into segments of 
approximately equal size. One segment was randomly selected, and households within it were systematically selected at a rate estimated to provide the needed sample size for the least populous risk group (PSAC). In households already selected for PSAC, SAC and adults were eligible for the survey sample. All members of the indicated risk-group(s) in a household were eligible.

Trained survey teams used Android-based smart phones running an Open Data Kit application to administer standardized survey questionnaires and collect geographic coordinates at each selected household. Data collection occurred between August and October 2017, approximately 5 months after the last STH MDA and approximately 1 month before the next one. During data collection, data issues were identified and addressed daily.

\section{Sample collection and laboratory procedures}

Plastic stool containers, without preservative, were left at selected households overnight and collected the next morning. Survey teams instructed participants on sample collection, emphasizing depositing stool samples the morning of collection. After collection, samples were stored in coolers and transported to field laboratories within $2 \mathrm{~h}$. Upon arrival, medical technologists processed samples by conducting dual-slide microscopy using the Kato-Katz technique [11]. A laboratory supervisor consulted on discordant results and independently reviewed $10 \%$ of samples.

\section{Data analysis}

De-identified data were stored on a secure cloud-based platform and analyzed using Stata version 15 (StataCorp. 2017. Stata Statistical Software: Release 15. College Station, TX) while accounting for the cluster sample survey design. All estimates include two-sided 95\% confidence intervals except for district-level prevalence which are designed to show the lowest WHO threshold achieved by calculating the upper, one-sided $95 \%$ confidence limit. A Chi-square $\left(x^{2}\right)$ test was used to test for significant differences in prevalence. $P$-values were considered significant if $<0.05$. Intensity of infection was determined according to WHO-defined classifications (Table 1). Missing data were typically addressed during data collection; however, if data were missing after data collection, they were excluded from analysis.

Table 1 WHO classifications of STH infection intensity (eggs per gram of stool) [2]

\begin{tabular}{llll}
\hline Organism & $\begin{array}{l}\text { Light intensity } \\
\text { infection }\end{array}$ & $\begin{array}{l}\text { Moderate intensity } \\
\text { infection }\end{array}$ & $\begin{array}{l}\text { Heavy intensity } \\
\text { infection }\end{array}$ \\
\hline A. lumbricoides & $<5000$ & $5000-49,999$ & $\geq 50,000$ \\
T. trichiura & $<1000$ & $1000-9999$ & $\geq 10,000$ \\
Hookworm & $<2000$ & $2000-3999$ & $\geq 4000$ \\
\hline
\end{tabular}

The authors generated all maps using ArcGIS 10.5 (Environmental Systems Research Institute, Redlands, CA). A central location within the cluster was selected for representation on maps.

\section{Results \\ Enrollment and sampling rates}

In the four surveyed districts, 7164 participants were enrolled and completed the questionnaire. Among those enrolled, 4361 (61\%) were female. Ages ranged from 1 to 80 years. A total of $4321(60.3 \%)$ provided a stool sample (Table 2), and among those, 4318 provided enough stool for testing. Insufficient training and supervision led to a failure to record the number of eligible participants within each household that were either unwilling or unable to participate; however, the overall non-response rate among enrolled persons was $39.7 \%(n=2843)$. It was $42.3 \%$ among PSAC, $40.2 \%$ among SAC, and $36.0 \%$ among adults. Among adults, males constituted only $10 \%$ of those enrolled and $8.5 \%$ of those who provided a sample. Adult males were frequently unavailable during the daytime when survey teams were present.

\section{STH prevalence}

The upper, one-sided 95\% confidence limit for prevalence of any STH was $<10 \%$ among all risk groups in Joypurhat, Chapai Nawabganj, and Rajshahi districts. The STH point prevalence in each risk group was significantly higher $(p<0.001)$ in Sirajganj District than other districts. District-level STH prevalence ranged from 3.3\% (upper, one-sided, 95\% confidence limit = $5.0 \%$ ) among adults in Joypurhat District to $29.1 \%$ (upper, one-sided, 95\% confidence limit $=36.0 \%$ ) among PSAC in Sirajganj District (Fig. 2).

Risk group-specific STH prevalence estimates were similar across Chapai Nawabganj, Joypurhat, and Rajshahi districts ranging from 3.3 to $5.4 \%$. Risk group prevalence in Sirajganj District ranged from 23.4 to $29.1 \%$ and was significantly higher than in other districts (all p-values $<0.001$ ).

\section{Moderate-to-high intensity infection}

Chapai Nawabganj and Joypurhat districts each had one participant with MHII. No MHII was detected in Rajshahi District. In Sirajganj District, MHII was 8.1\% (95\% CI: $4.8-13.2 \%$ ), $6.6 \%$ (95\% CI: $3.4-12.7 \%$ ), and $8.1 \%$ (95\% CI, 4.4-14.4\%) among PSAC, SAC, and adults respectively (Fig. 3). Risk group-specific MHII estimates in Sirajganj District were significantly higher than other districts (all p-values $<0.001$ ) and well above the threshold for elimination as a public health problem $(<1 \%)$. The percentage of MHII and PC coverage by risk group are shown for Sirajganj District in Fig. 3. 
Table 2 Enrollment and sampling rates among PSAC, SAC, and adults by district

\begin{tabular}{|c|c|c|c|c|c|c|c|c|}
\hline \multirow[b]{3}{*}{ District } & \multicolumn{2}{|c|}{$\begin{array}{l}\text { PSAC (1-4 years) } \\
\mathbf{n}=\mathbf{2 4 4 8}\end{array}$} & \multicolumn{2}{|c|}{$\begin{array}{l}\text { SAC (5-14 years) } \\
\boldsymbol{n}=\mathbf{2 6 1 4}\end{array}$} & \multicolumn{2}{|c|}{$\begin{array}{l}\text { Adults ( } \geq 15 \text { years) } \\
\boldsymbol{n}=\mathbf{2 1 0 2}\end{array}$} & \multicolumn{2}{|l|}{$\begin{array}{l}\text { Total } \\
N=7164\end{array}$} \\
\hline & Enrolled $^{a}$ & $\begin{array}{l}\text { Provided } \\
\text { Sample }\end{array}$ & Enrolled $^{a}$ & $\begin{array}{l}\text { Provided } \\
\text { Sample }\end{array}$ & Enrolled $^{a}$ & $\begin{array}{l}\text { Provided } \\
\text { Sample }\end{array}$ & Enrolled $^{a}$ & $\begin{array}{l}\text { Provided } \\
\text { Sample }\end{array}$ \\
\hline & $n$ & $n(\%)$ & $n$ & $n(\%)$ & $n$ & $n(\%)$ & $n$ & $n(\%)$ \\
\hline \multicolumn{9}{|c|}{ Chapai Nawabganj } \\
\hline Male & 318 & $170(53.5)$ & 357 & $207(58.0)$ & 29 & $14(48.3)$ & 704 & $391(55.5)$ \\
\hline Female & 303 & $175(57.8)$ & 329 & $190(57.8)$ & 451 & $299(66.3)$ & 1083 & $664(61.3)$ \\
\hline \multicolumn{9}{|l|}{ Joypurhat } \\
\hline Male & 281 & $190(67.6)$ & 306 & $179(58.5)$ & 120 & $68(56.7)$ & 707 & $437(61.8)$ \\
\hline Female & 250 & $138(55.2)$ & 329 & $196(59.6)$ & 462 & $300(64.9)$ & 1041 & $634(60.9)$ \\
\hline \multicolumn{9}{|l|}{ Rajshahi } \\
\hline Male & 352 & $201(57.1)$ & 308 & $204(66.2)$ & 29 & $17(58.6)$ & 689 & $422(61.2)$ \\
\hline Female & 285 & $168(58.9)$ & 320 & $179(55.9)$ & 496 & $314(63.3)$ & 1101 & $661(60.0)$ \\
\hline \multicolumn{9}{|l|}{ Sirajganj } \\
\hline Male & 342 & 205 (59.9) & 328 & $201(61.3)$ & 33 & $15(45.5)$ & 703 & $421(59.9)$ \\
\hline Female & 317 & $166(52.4)$ & 337 & $206(61.1)$ & 482 & 319 (66.2) & 1136 & $691(60.8)$ \\
\hline
\end{tabular}

\section{Parasite type}

In all districts, $A$. lumbricoides was the most common STH parasite $[8.2 \%(n=352)]$, followed by T. trichiura $[0.9 \%(n=$ 37)] and hookworm [0.6\% $(n=27)]$. A. lumbricoides was most prevalent among SAC [8.9\% $(n=139)]$, followed by PSAC [8.1\% $(n=114)]$ and adults $[7.4 \%(n=99)]$. All polyparasitism was due to A. lumbricoides and T. trichiura [0.3\% $(n=14)]$ and only identified in Sirajganj District. Prevalence of each species was consistent across risk groups (Fig. 4). Hookworm was the least common infection (adults $n=12$, SAC $n=7$, PSAC $n=8$ ). No significant differences in STH parasite type were observed across districts.

\section{Geographic distribution of STH}

Within and across districts, the geographic distribution of STH prevalence varied. Due to the geographic distribution

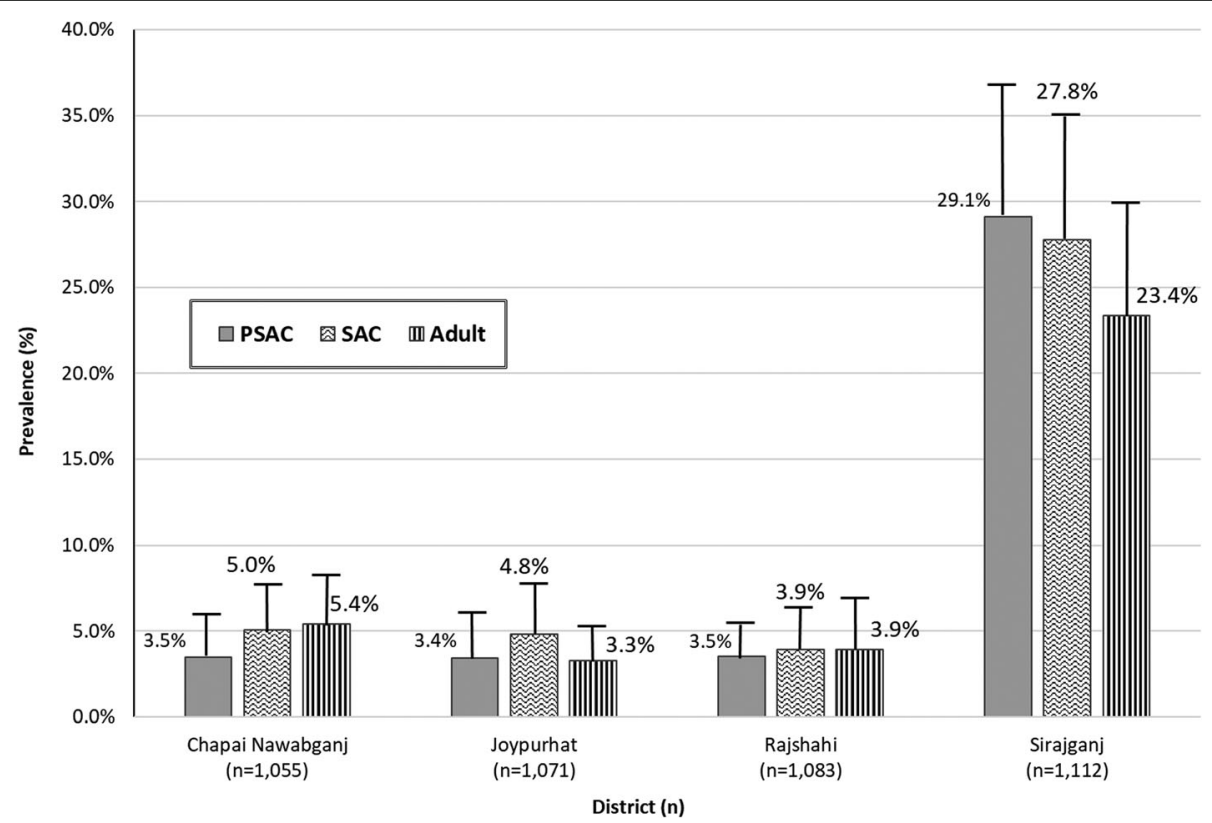

Fig. 2 Prevalence (upper, one-sided, 95\% confidence limit) of any STH by risk group and district 


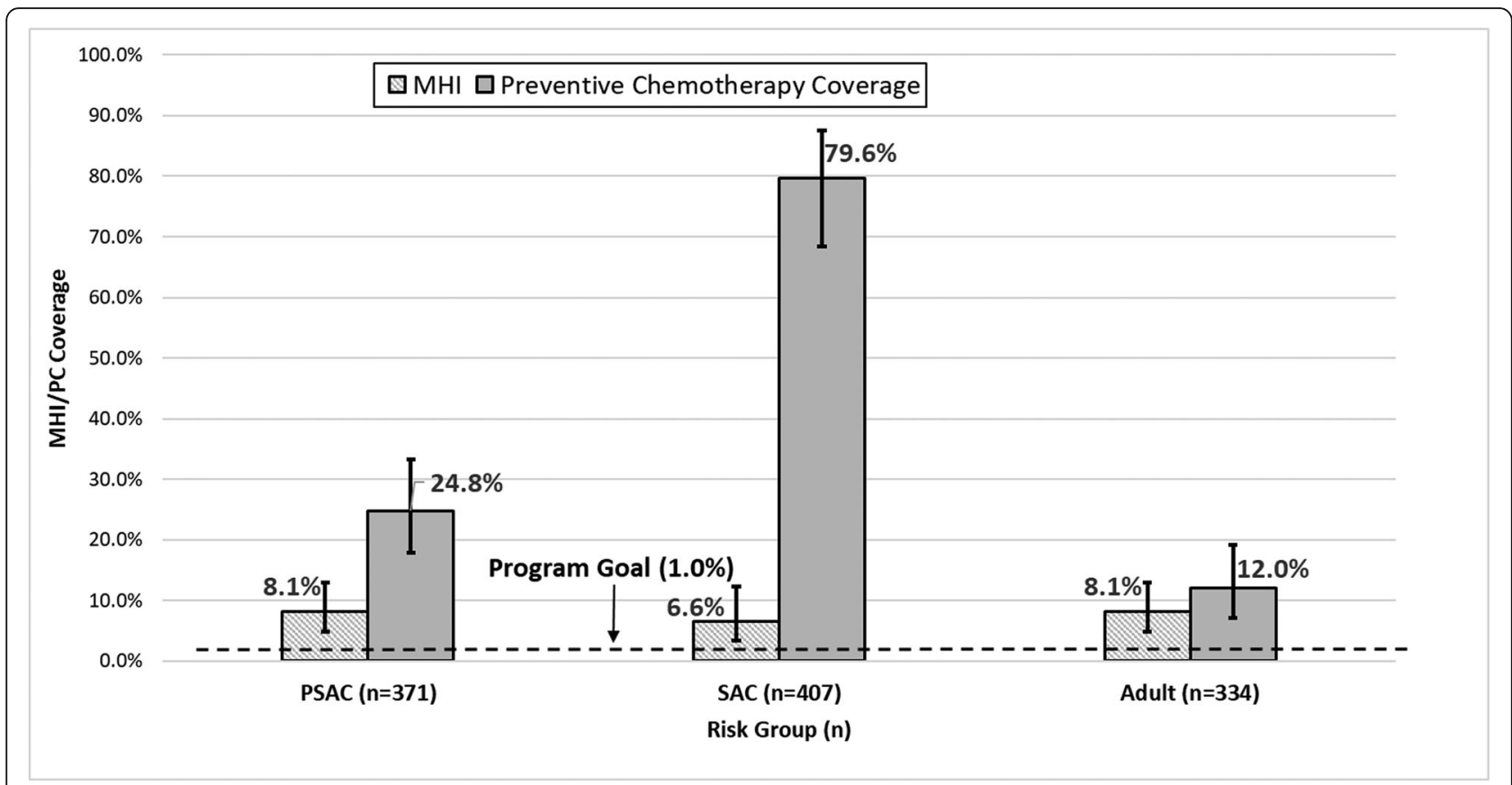

Fig. 3 Any STH MHII and PC coverage $(95 \%$ Cl) by risk group, Sirajganj District

and high prevalence in Sirajganj District, we mapped overall prevalence and PC coverage (Fig. 5). Sirajganj subdistricts of concern included Belkuchi (SAC: $57.1 \%$ prevalence, 20.0\% MHII) and Kamarkhanda (SAC: 50.0\% STH prevalence, $28.6 \%$ MHII). Belkuchi had the highest STH prevalence in PSAC $[60.5 \%(n=23)]$, SAC $[57.1 \%(n=$ $20)]$, and adults $[50.0 \%(n=15)]$ (Table 3). Belkuchi had the highest MHII among PSAC [23.7\% $(n=9)]$ and adults [20.0\% $(n=6)]$, and Kamarkhanda had the highest MHII among SAC. Three sub-districts had no MHII (Chauhali,
Kazipur, and Tarash) and STH prevalence in those subdistricts ranged from 3.2\% $(\mathrm{n}=1)$ among adults in Kazipur to $42.9 \%(\mathrm{n}=9)$ among SAC in Chauhali.

Preventive chemotherapy coverage and water, sanitation and hygiene (WASH) indicators

In all districts, self-reported ICSPM PC coverage for SAC was above the MOHFW target of $\geq 75 \%$ [2] (range from 79.6 to $89.8 \%$ ). However, ICSPM PC coverage was

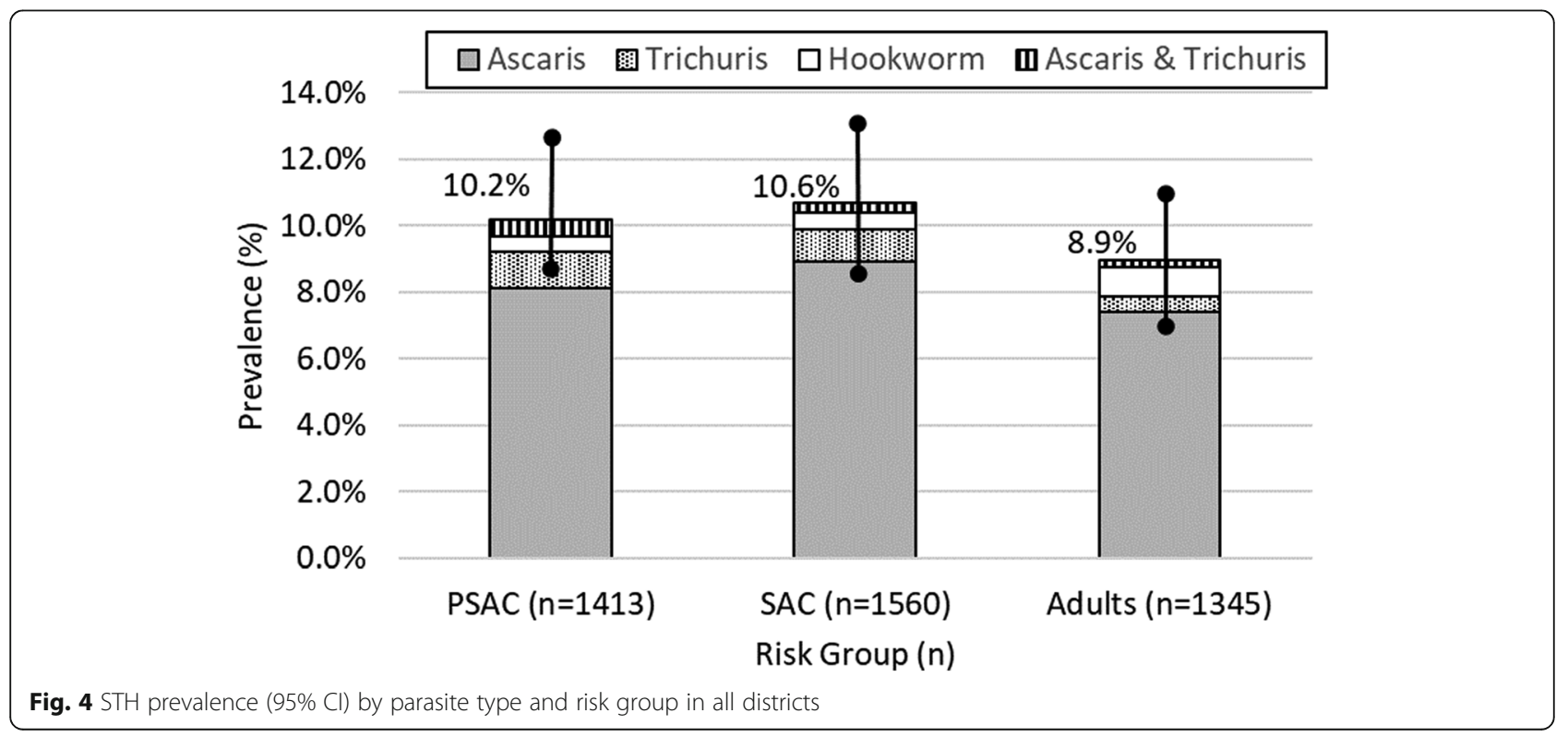




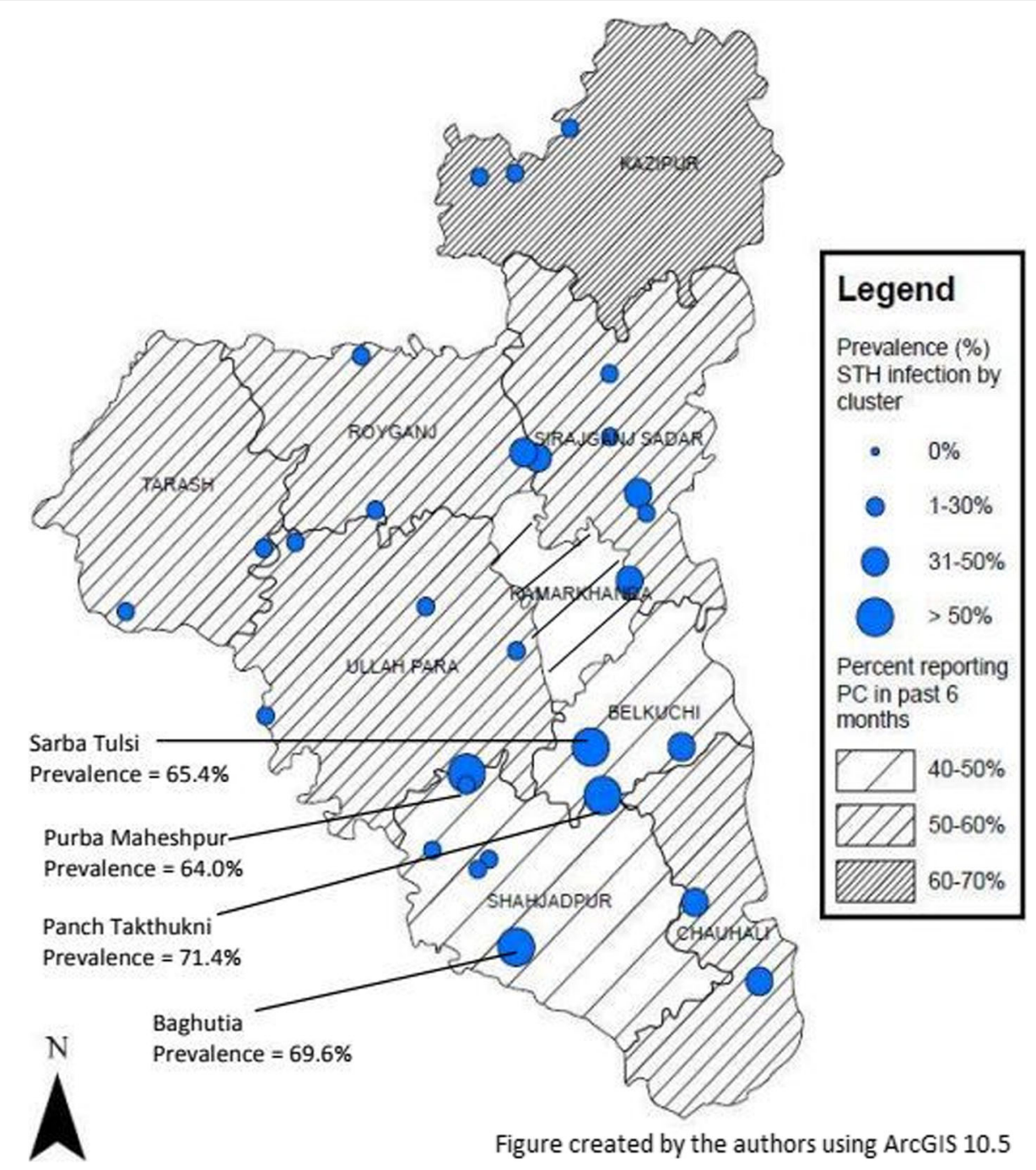

Fig. 5 Geographic distribution of STH prevalence and PC coverage in all risk groups combined, Sirajganj District

significantly lower in PSAC [overall $=32.6 \%(\mathrm{p}<0.001)$ ] and adults [overall $=19.5 \%(\mathrm{p}<0.001)$ ] than in SAC.

We also compared ICSPM PC coverage among SAC to SAC coverage reported by the MOHFW as part of routine program monitoring. MOHFW-reported coverage was from the MDA preceding the ICSPM [MOHFW unpublished data, 2017 (data not shown)]. Reported coverage data for other risk groups were unavailable. ICSPM PC coverage in SAC was lower, in some districts substantially, than MOHFW-reported coverage. ICSPM and reported coverage differed noticeably in Chapai Nawabganj (80.6 and 100\%, respectively) and Sirajganj District (79.6 and 95.5\%, respectively). ICSPM results across districts showed the most common source of deworming drugs for SAC was school-based MDA, while purchasing PC was the most common source for PSAC and adults (Table 4).
Improved sanitation at home, school, or work was over $90 \%$ in all districts. The prevalence of improved flooring ranged from 10.6\% (95\% CI: 8.9-12.6\%) in Sirajganj District to $34.7 \%$ (95\% CI: $24.5-46.5 \%$ ) in Chapai Nawabganj District. The presence of a handwashing station with soap in the home was observed in $<50 \%$ of households in all districts. Sirajganj District had the lowest observed household coverage of handwashing stations with soap (36.2\%; 95\% CI: 21.1-54.7\%); however, compared to the other districts the difference was not statistically significant (Table 5).

\section{Discussion}

Comprehensive, community-based surveys such as the ICSPM are intended to support STH program monitoring, particularly for advanced programs that have delivered interventions for $\geq 5$ years. WHO recommends that programs assess, using prevalence ranges, possible 


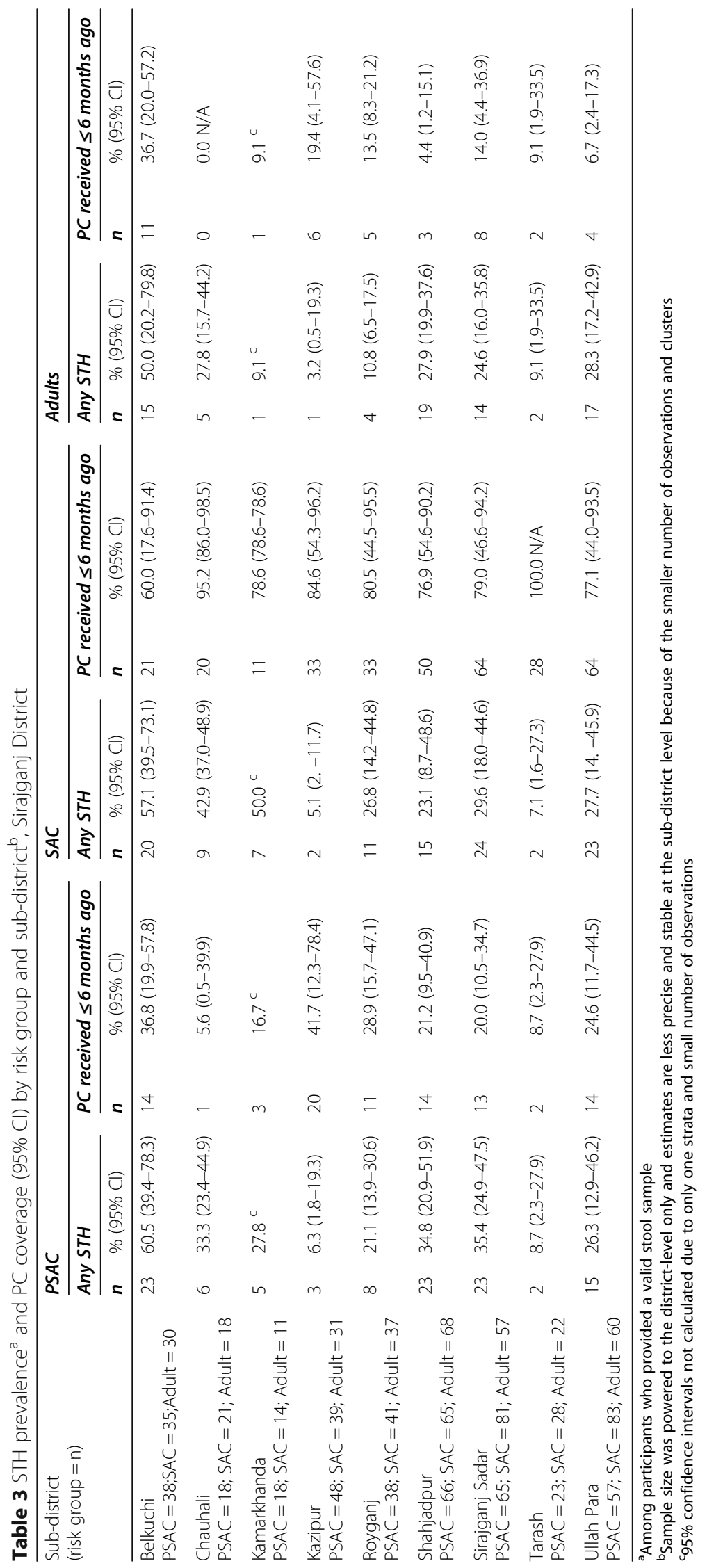


Table 4 Preventive chemotherapy coverage and most common source of PC (95\% Cl) by risk group and district

\begin{tabular}{|c|c|c|c|c|c|c|}
\hline & \multicolumn{2}{|c|}{ PSAC } & \multicolumn{2}{|l|}{$S A C$} & \multicolumn{2}{|c|}{ Adults } \\
\hline & $n$ & $\%[95 \% \mathrm{Cl}]$ & $n$ & $\%[95 \% \mathrm{Cl}]$ & $n$ & $\%[95 \% \mathrm{Cl}]$ \\
\hline District & \multicolumn{6}{|c|}{$P C$ received $\leq 6$ months ago } \\
\hline Chapai Nawabganj & 152 & $44.1[35.1-53.4]$ & 320 & $80.6[69.3-88.4]$ & 65 & $20.8[15.3-27.5]$ \\
\hline Joypurhat & 112 & $34.1[26.2-43.1]$ & 324 & 86.3 [77.8-91.9] & 93 & $25.3[20.0-31.5]$ \\
\hline Rajshahi & 104 & $28.2[20.0-38.1]$ & 344 & $89.8[82.2-94.4]$ & 64 & $19.3[13.0-27.8]$ \\
\hline Sirajganj & 92 & $24.8[17.9-33.3]$ & 324 & $79.6[68.4-87.6]$ & 40 & $12.0[7.2-19.2]$ \\
\hline District & \multicolumn{6}{|c|}{ Most common source of $P C^{a}$} \\
\hline Chapai Nawabganj & 192 & Purchased 86.1 [78.8-91.1] & 347 & School 90.1 [84.6-93.8] & 150 & Purchased 80.2 [71.7-86.6] \\
\hline Joypurhat & 110 & Purchased 60.8 [45.3-74.3] & 319 & School 89.0 [83.3-93.0] & 182 & Purchased 83.9 [73.7-90.6] \\
\hline Rajshahi & 146 & Purchased 80.2 [71.4-86.8] & 353 & School 93.4 [88.4-96.3] & 168 & Purchased 74.3 [62.1-83.7] \\
\hline Sirajganj & 177 & Purchased 94.7 [88.7-97.6] & 324 & School 82.4 [73.0-89.1] & 190 & Purchased 90.9 [82.0-95.6] \\
\hline
\end{tabular}

"Survey question: "The last time you swallowed deworming medication, where did you receive it?"

changes to MDA frequency following 5-6 years of MDAs [5]. Given ICSPM results on prevalence and PC and sanitation coverage, as well as 20 completed MDA rounds, the MOHFW will pilot stopping the twiceannual STH MDAs in selected areas within Joypurhat, Rajshahi, and Chapai Nawabganj districts. WHO recommends MDAs once every 2 years in areas with $\geq 2 \%$ and $<10 \%$ prevalence [4]. However, the MOHFW is cautiously approaching the decision given potential disease recrudescence and the lack of established surveillance for STH. Other national programs likely have similar concerns. Further guidance to national programs on monitoring STH in low-prevalence districts is warranted, including on methods for parasitologic assessments or surveillance if MDAs cease. Outside of pilot areas, the MOHFW will continue twice-annual MDAs. The MOHFW will consider sanitation coverage in selecting pilot areas. Although MOHFW does not deliver WASH interventions, WASH partners could use ICSPM data to geographically target interventions. Additionally, the low prevalence of improved household flooring (10.6\%) and of home handwashing stations with soap (36.2\%) highlights the need for behavioral interventions in Sirajganj District.

Joypurhat, Rajshahi, and Chapai Nawabganj districts are approaching elimination of STH as a public health problem, with MHII across risk groups of $<10 \%$. However, the MOHFW does not currently plan to assess whether the $1 \%$ "elimination" target has been met. The high cost required to obtain an adequate survey sample size is currently prohibitive. Furthermore, existing WHO guidance uses prevalence of any STH instead of MHII to determine MDA frequency. Limited available resources could be better used given the lack of a clear action tied to reaching the "elimination" target.

Similar to our results, high STH prevalence has been noted to be spatially focalized in Kenya [12], Burkina Faso [13], and Honduras [14]. Additionally, a recent systematic review in Asia found substantial heterogeneity in the geographic distribution of STH between and within countries [15]. Targeted programmatic focus by the MOHFW on high prevalence areas should improve overall intervention efficiency and impact. ICSPM results identified Sirajganj District for such enhanced control efforts. In Sirajganj, low PC coverage, particularly among PSAC (24.8\%), likely contributes to high STH prevalence and MHII. Among PSAC who received PC in the district, 94.7\% relied on purchasing the drug, indicating the need to improve MOHFW efforts to reach this risk group. Bangladesh has experienced inconsistent drug availability for PSAC partly due to procurement challenges. MDAs for SAC use drug donated through WHO, while there is no available donation for PSAC. Regardless of the impressive PC coverage of SAC, the MOHFW is unlikely to eliminate STH as a public health problem with the generally low coverage of

Table 5 Sanitation and hygiene indicators (95\% Cl) by district

\begin{tabular}{|c|c|c|c|c|}
\hline & $\begin{array}{l}\text { Chapai Nawabganj } \\
n=1055\end{array}$ & $\begin{array}{l}\text { Joypurhat } \\
n=1068\end{array}$ & $\begin{array}{l}\text { Rajshahi } \\
n=1083\end{array}$ & $\begin{array}{l}\text { Sirajganj } \\
n=1112\end{array}$ \\
\hline WASH Indicator & $\%[95 \% \mathrm{Cl}]$ & $\%[95 \% \mathrm{Cl}]$ & $\%[95 \% \mathrm{Cl}]$ & $\%[95 \% \mathrm{Cl}]$ \\
\hline Improved sanitation ${ }^{a}$ (home) & $97.6[94.4-99.0]$ & $94.0[84.7-97.8]$ & $98.3[94.7-99.5]$ & $95.3[89.6-98.0]$ \\
\hline Handwashing station with soap (home) & $42.3[26.4-60.0]$ & $42.6[26.8-60.1]$ & $44.5[28.6-61.6]$ & $36.2[21.1-54.7]$ \\
\hline
\end{tabular}

${ }^{\mathrm{a}}$ Improved sanitation defined as: flush toilet to sewer/septic; ventilated improved pit latrine; pit latrine w/ slab; or composting toilet 
PSAC. In surveyed districts, $44.1 \%$ was the highest district-level PSAC coverage.

The discrepancy between self-reported ICSPM SAC PC coverage and MOHFW-reported SAC PC coverage in Sirajganj District (79.6\%. and 95.5\%, respectively) suggests over-reporting in routine MOHFW monitoring of MDAs, hindering the timely addressing of implementation challenges. Recall bias could contribute to differing coverage figures. Additional PC coverage assessments will benefit the program, and enhanced MOHFW supervision during MDAs will be a priority going forward.

In Sirajganj, enhanced MOHFW supervision will further MOHFW efforts to identify and address programmatic gaps. Increased supervision, from national, division, and district personnel, will build capacity of local personnel through one-on-one training. Increased supervision will also improve adherence to established program protocols. In Sirajganj, sub-districts with markedly higher STH prevalence warrant attention.

WHO has recommended PC for all non-pregnant WRA "living in areas" with baseline STH prevalence $\geq 20 \%$ among WRA [3]. The MOHFW is considering where and how to apply this guidance. In Bangladesh, there are no baseline data for WRA. ICSPM data could fill this need. However, it would require surveying the remaining districts in the country, and the required resources are unavailable. In ICSPM-surveyed districts that have $\geq 20 \%$ prevalence among WRA, the MOHFW has a sound basis for expanding administration of $\mathrm{PC}$ to this risk group. Given the low prevalence in three of four ICSPM districts, treating 46.6 million WRA nationwide is unwarranted. Again, there are important implications for having statistically valid district-level data.

As the MOHFW considers WHO guidance on PC for WRA, notably there was low hookworm prevalence $(<$ $0.7 \%$ overall). Hookworm infections are of concern to that risk group because of resulting anemia. While our surveys relied on Kato-Katz testing, with its challenges in detecting hookworm in samples more than a few hours old [16], the lack of hookworm infections is consistent with past surveys in Bangladesh among children [17-21] and WRA [22]. Furthermore, a 2018 systematic review on STH combined the results of seven studies $(n=2886)$ from Bangladesh and estimated prevalence of hookworm to be only $3 \%$. Given the program goal, the lack of hookworm and T. trichiura infections means that treating WRA, at least nationwide, would unnecessarily divert resources from the main STH parasite and the population it impacts: A. lumbricoides in children [23].

National programs in resource-limited countries, such as Bangladesh, have often relied on school-based parasitologic monitoring of STH. Unlike WHOrecommended school-based surveys, the ICSPM provides the MOHFW the necessary data to understand program status at the implementation unit (district) level. Because the MOHFW relies on district public health and education systems to deliver interventions and monitor PC coverage, it follows that assessment of reported PC coverage and STH epidemiology at the district level is useful for program managers. Assessing the impact of control efforts in a district also provides critical guidance to district health and education offices.

\section{Limitations}

For practicality and cost, the surveys were designed to assess STH prevalence by WHO prevalence range to a threshold of $<10 \%$. Notably, data from multiple districts could be combined to reach a sample size that would have adequate statistical power for assessing the $\leq 1 \%$ MHII goal, but this could result in modifying PC frequency without accounting for heterogeneity in the geographic distribution of STH.

Non-response was almost $40 \%$ across surveyed districts. There was a large disparity in participation among adult males and females, both in those enrolled, and those who provided a sample. In future ICSPMs, the MOHFW plans to address high non-response by improving coordination with local health authorities and increasing pre-survey community mobilization. The MOHFW is considering whether it is logistically and financially feasible, during future ICSPMs, to make follow-up visits to relevant households. As discussed, recall bias could have led to inaccurate ICSPM PC coverage estimates. Finally, although participants were asked to provide stool samples in the morning, substantial delays between stool deposit and examination were possible. Such delays may have led to the underestimation of hookworm prevalence.

\section{Conclusion}

The ICSPM provided a needed, innovative approach to assess programmatic progress. The surveys highlighted areas where high prevalence persists, despite 20 MDA rounds, and that require enhanced monitoring and intervention delivery. ICSPMs require more resources than school-based parasitologic surveys; however, the resources used in Bangladesh were comparable to similar surveys (e.g. LF Transmission Assessment Surveys, Trachoma district mapping), and ICSPMs serve multiple purposes and achieve important efficiencies.

Documenting the impact of years of PC will help focus program resources on areas of persistent high transmission, as in Sirajganj. The resources freed up by a geographically-targeted approach could meet other needs including establishing ongoing parasitologic monitoring in low prevalence districts and improving PC coverage among risk groups other than SAC. 


\section{Abbreviations}

ICSPM: Integrated Community-based Survey for Program Monitoring: LF: Lymphatic filariasis; MDA: Mass drug administration; MHII: Moderate-tohigh intensity infection of STH; MOHFW: Ministry of Health \& Family Welfare, Government of Bangladesh; PC: Preventive chemotherapy; PSAC: Preschoolage children; SAC: School-age children; STH: Soil-transmitted helminthiasis; WASH: Water, sanitation, and hygiene; WHO: World Health Organization; WRA: Women of reproductive age

\section{Acknowledgments}

Implementation of the surveys would not have been possible without the dedicated staff of the national STH control program of Bangladesh, district health officials, and upazila health officers as well as the people who participated in the surveys. The work was made possible through the generous financial support of Nutrition International (with funding from the Government of (anada) and Johnson \& Johnson.

\section{Authors' contributions}

All co-authors have reviewed and approved the submitted version of the manuscript. SLD analyzed and interpreted the data and drafted and revised the work. AHJ helped conceptualize the study, acquire, analyze and interpret the data, and draft and revise the work. ST acquired the data and revised the work. AAK helped conceptualize the study, acquire the data, and revise the work. AJ helped acquire the data and revise the work. SIZ helped acquire the data and edit the work. MMR helped acquire the data. BMM helped conceptualize the study, acquire, analyze and interpret the data, and revise the work. MSD conceptualized the study, interpreted the data, and drafted and revised the work. RIT interpreted the data and drafted and revised the work. MJK helped conceptualize the study, acquire, interpret the data, and revise the work.

\section{Funding}

The work was conducted with financial support from Nutrition International through funding from the Government of Canada. Johnson \& Johnson also provided financial support for this work. The funders of this work had no role in the design of the study, data collection, data analysis, or data interpretation. The funders had no role in writing the manuscript.

\section{Availability of data and materials}

The datasets generated for and analyzed in the study are not publicly available but are available from the corresponding author upon reasonable request.

\section{Ethics approval and consent to participate}

The survey protocol was reviewed and approved by the Bangladesh Medical Research Council Institutional Review Board (BMRC/NREC/2016-2019/274) including use of verbal consent and assent. The IRB approved verbal consent because it considered the survey low risk and part of the routine program monitoring of STH control activities. Informed consent or assent was obtained from all participants.

\section{Consent for publication}

Not applicable.

\section{Competing interests}

The authors declare that they have no competing interests.

\section{Author details}

'Children Without Worms, The Task Force for Global Health, 325 Swanton Way, Decatur, GA, USA. ${ }^{2}$ Communicable Disease Control Department, Directorate General of Health Services, Ministry of Health and Family Welfare, Road \# 29, New DOHS, Mohakhali, Dhaka, Bangladesh. ${ }^{3}$ Elimination of Lymphatic Filariasis \& STH Control Program, Directorate General of Health Services, Ministry of Health \& Family Welfare, Road \# 29, New DOHS, Mohakhali, Dhaka, Bangladesh. ${ }^{4}$ Mahidol Oxford Tropical Medicine Research Unit, Faculty of Tropical Medicine, Mahidol University, C/O DOHS, Road \# 29, New DOHS, Mohakhali, Dhaka, Bangladesh.
Received: 25 October 2019 Accepted: 22 April 2020

Published online: 12 May 2020

\section{References}

1. Pullan RL, Smith JL, Jasrasaria R, Brooker SJ. Global numbers of infection and disease burden of soil transmitted helminth infections in 2010. Parasites Vector. 2014;7:1.

2. World Health Organization. Soil-transmitted helminthiases: Eliminating soiltransmitted helminthiases as a public health problem in children: Progress report 2001-2010 and strategic plan 2011-2020. Geneva; 2012.

3. World Health Organization. Guideline: preventive chemotherapy to control soil-transmitted helminth infections in at-risk population groups. Geneva; 2017.

4. World Health Organization. Assessing the Epidemiology of Soil-transmitted Helminths during a Transmission Assessment Survey in the Global Programme for the Elimination of Lymphatic Filariasis. Geneva: World Health Organization; 2015.

5. World Health Organization. Helminth control in school-aged children: A guide for managers of control programmes. Geneva; 2011.

6. PCT Databank. World Health Organization, Geneva. 2018. Accesed 1 Mar 2019.

7. Shamsuzzaman AKM, Haq R, Karim MJ, Azad MB, Mahmood ASMS, Khair A, et al. The significant scale up and success of transmission assessment surveys 'TAS' for endgame surveillance of lymphatic filariasis in Bangladesh: one step closer to the elimination goal of 2020. PLoS Negl Trop Dis. 2017; 11:1.

8. World Health Organization. Lymphatic filariasis: monitoring and epidemiological assessment of mass drug administration - A manual for national elimination programmes. Geneva; 2011. p. 79.

9. World Health Organization. Assessing the Epidemiology of Soil-transmitted Helminths during a Transmission Assessment Survey in the Global Programme fro the Elimination of Lymphatic Filariasis. Geneva; 2015. p. 42.

10. Bangladesh Bureau of Statistics. Population \& Housing Census 2011. Dhaka; 2011.

11. Katz N, Chaves A, Pellegrino J. A simple device for quantitative stool thicksmear technique in Schistosomiasis mansoni. Rev Inst Med Trop Sao Paulo. 1972;14:6.

12. Smith JL, Sturrock HJW, Assefa L, Nikolay B, Njenga SM, Kihara J, et al. Factors associated with the performance and cost-effectiveness of using lymphatic Filariasis transmission assessment surveys for monitoring soil-transmitted Helminths: a case study in Kenya. Am J Trop Med Hyg. 2015;92:2.

13. Drabo F, Ouedraogo H, Bougma R, Bougouma C, Bamba I, Zongo D, et al. Successful control of soil-transmitted Helminthiasis in school age children in Burkina Faso and an example of community-based assessment via lymphatic Filariasis transmission assessment survey. PLoS Negl Trop Dis. 2016;10:5

14. Sanchez AL, Gabrie JA, Rueda MM, Mejia RE, Bottazzi ME, Canales M. A scoping review and prevalence analysis of soil-transmitted helminth infections in Honduras. PLoS Negl Trop Dis. 2014;8:1.

15. Silver ZA, Kaliappan SP, Samuel P, Venugopal S, Kang G, Sarkar R, et al. Geographical distribution of soil transmitted helminths and the effects of community type in South Asia and South East Asia - a systematic review. PLoS Negl Trop Dis. 2018;12:1.

16. Dacombe RJ, Crampin AC, Floyd S, Randall A, Ndhlovu R, Bickle Q, et al. Time delays between patient and laboratory selectively affect accuracy of helminth diagnosis. Trans R Soc Trop Med Hyg. 2007;101:2.

17. Kongsbak K, Wahed MA, Friis H, Thilsted SH. Acute-phase protein levels, diarrhoea, Trichuris trichiura and maternal education are predictors of serum retinol: a cross-sectional study of children in a Dhaka slum, Bangladesh. Brit J Nutr. 2007;96:725-34.

18. Northrop-Clewes CA, Rousham EK, Mascie-Taylor CN, Lunn PG. Anthelmintic treatment of rural Bangladeshi children: effect on host physiology, growth, and biochemical status. Am J Clin Nutr. 2001;73(1):53-60.

19. Hawlader M, Ma E, Noguchi E, Itoh M, Arifeen S, Persson L, et al. Ascaris lumbricoids infection as a risk factor for asthma and atopy in rural Bangladeshi children. Trop Med Health. 2014;42(2):77-85.

20. Lin A, Arnold BF, Afreen S, Goto R, Huda TM, Haque R, et al. Household environmental conditions are associated with enteropathy and impaired growth in rural Bangladesh. Am J Trop Med Hyg. 2013;89(1):130-7.

21. Persson V, Ahmed F, Gebre-Medhin M, Greiner T. Increase in serum betacarotene following dark green leafy vegetable supplementation in 
Mebendazole-treated school children in Bangladesh. Eur J Clin Nutr. 2001; 55(1):1-9.

22. Ziauddin Hyder $\mathrm{S}$, et al. Anaemia among non-pregnant women in rural Bangladesh. Public Health Nurt. 2001:4(1):79-83.

23. de Silva N, Chan M, Bundy D. Morbidity and mortality due to ascariasis: reestimation and sensitivity analysis of global numbers at risk. Tropical Med Int Health. 1997;2(6):513-8.

\section{Publisher's Note}

Springer Nature remains neutral with regard to jurisdictional claims in published maps and institutional affiliations.

Ready to submit your research? Choose BMC and benefit from:

- fast, convenient online submission

- thorough peer review by experienced researchers in your field

- rapid publication on acceptance

- support for research data, including large and complex data types

- gold Open Access which fosters wider collaboration and increased citations

- maximum visibility for your research: over $100 \mathrm{M}$ website views per year

At $\mathrm{BMC}$, research is always in progress. 